\title{
Recomendaciones de organización, características y funcionamiento en Servicios o Unidades de Neonatología
}

\author{
DIRECTIVA RAMA DE NEONATOLOGÍA DE LA SOCIEDAD CHILENA DE PEDIATRÍA: \\ José M. Novoa P. ${ }^{1}$, Marcela Milad A. ${ }^{2}$, Guillermo Vivanco G. ${ }^{3,4}$, Jorge Fabres B. ${ }^{3,5}$ y Rodrigo Ramírez F.,6 \\ 1. Presidente Rama de Neonatología, Jefe de Neonatología Hospital Padre Hurtado. Profesor Departamento de Pediatría, \\ Facultad de Medicina Universidad del Desarrollo-Clínica Alemana. \\ 2. Vice-Presidente Rama de Neonatología, Jefe de Neonatología Clínica Santa María. Docente Departamento de Pediatría, \\ Universidad de Los Andes. \\ 3. Director Rama de Neonatología. \\ 4. Jefe de Neonatología Hospital Félix Bulnes. Profesor Titular de Neonatología. Universidad Mayor. \\ 5. Neonatólogo, Servicio de Neonatología Hospital Clínico Universidad Católica de Chile. Profesor Asistente de Pediatría, \\ Departamento de Pediatría. Pontificia Universidad Católica de Chile. \\ 6. Neonatólogo, Servicio de Neonatología Hospital Clínico Universidad de Chile. Profesor Asistente de Pediatría \\ Departamento de Obstetricia y Ginecología. Universidad de Chile.
}

\section{Prólogo}

Las cifras de mortalidad infantil en Chile indiscutiblemente han mejorado a través de los años. La disminución de la mortalidad neonatal es fundamental para lograr este propósito, ya que representa dos tercios de la mortalidad registrada en el segmento infantil.

El Ministerio de Salud y las Sociedades Científicas constituyen una alianza estratégica para alcanzar los objetivos óptimos de salud de una población determinada. En este sentido, a lo largo de los años hemos constatado experiencias exitosas en el quehacer conjunto de la autoridad sanitaria y estas sociedades. La Neonatología no debería ser una excepción en este diseño de trabajo conjunto.

Valoramos enormemente la labor desarrollada por la Rama de Neonatología de la Sociedad Chilena de Pediatría, en orden a generar las recomendaciones expuestas en esta publicación, cuyo objetivo es que sirva como referente para la gestión neonatal y oriente la cartera de inversiones en una de las áreas más costosas del quehacer hospitalario.

Uno de los elementos más importantes del trabajo en salud, guarda relación con el ordenamiento del quehacer asistencial. El horizonte, es desarrollar un proceso de consensos, en que equipos clínicos y autoridades en salud trabajemos en disminuir la variabilidad en los proce- sos, lo que permitirá ver a su vez, mejorar resultados en oportunidad, calidad e impacto en nuestras acciones.

Uno de los avances logrados hasta ahora son los recursos inyectados al desarrollo de la Neonatología, a través de las patologías con garantías explícitas en salud (GES). Esto nos ha permitido ir acortando las brechas en la especialidad, sin embargo, sabemos que aún falta mucho, en este trabajo permanente en que los recursos son limitados en relación a las aspiraciones y al rápido avance de la medicina. Pero no todo es recursos económicos.

Este trabajo nos obliga a optimizar resultados especialmente si consideramos elementos de modernización en el trabajo, plasmados en muchos de los aspectos planteados en Organización, Sistema de Registro y Evaluación de Calidad.

Invitamos a los equipos de Salud Neonatal a considerar que estas recomendaciones sólo pueden llegar a tener éxito, en la medida en que todos los implicados trabajemos con seriedad, rigor, creatividad y respeto. Para ello debemos asumir cada uno de nuestros roles, no olvidando que el gran objetivo que nos une es la mejor atención a nuestros neonatos, sus familias y la comunidad.

Dr. Julio Montt Vidal

Sub-Secretario de Redes Asistenciales Ministerio de Salud. 


\section{Introducción}

La creación de Guías de Planificación para servicios o unidades de cuidados neonatales se remonta en la literatura médica al año 1976. Desde esa fecha hasta ahora, se han publicado numerosos trabajos de equipos de expertos, multidisciplinarios, destacando la Academia Americana de Pediatría, el Colegio Americano de Obstetricia y Ginecología y la Asociación Española de Neonatología, entre otros.

Desde el inicio de los años 80, en Chile se organizó el cuidado intensivo neonatal, creándose alrededor de 30 centros regionales públicos en las grandes ciudades, lo que originó un importante impacto en la mejoría de la sobrevida del recién nacido ( $\mathrm{RN}$ ) en las últimas décadas. Posteriormente a través de la incorporación de las patologías GES se ha logrado garantizar el uso de surfactante, indometacina, terapias respiratorias de alto costo en SDR, manejo de neonatos con cardiopatías, entre otras acciones. Por todo lo anterior, se ha logrado a través de los años una disminución significativa de la mortalidad neonatal en nuestro país, alcanzando cifras cercanas a los 5 por cada 1000 nacidos vivos. Sin embargo, aún es posible observar que existen diferencias significativas en los resultados obtenidos entre diferentes hospitales y clínicas privadas tanto de la Región Metropolitana como en regiones, mientras en algunos centros las cifras de mortalidad neonatal son mucho mayores al promedio Nacional, en otras son cercanas a la mitad.

Entre las principales causas de mortalidad neonatal destacan la prematurez extrema, las malformaciones congénitas, infecciones y problemas respiratorios complejos. En estos problemas, en especial en los prematuros de extremo bajo peso, existe en nuestro país una importante menor sobrevida comparado con las cifras de los países desarrollados. Estas diferencias observadas se deben probablemente a la desigual disponibilidad de recursos, tanto de personal entrenado como de equipamiento e infraestructura que permita entregar un tratamiento oportuno a estos pacientes complejos.

Numerosos trabajos en la literatura han demostrado los beneficios de la regionalización en nacimientos de alto riesgo. Especialmente en menores de 1000 gramos, la mortalidad es más baja en las unidades de mayor complejidad y mayor volumen de pacientes a pesar de ser centros de derivación de pacientes críticos. Entre centros de igual complejidad, la mortalidad es menor en aquellos que tienen mayor índice ocupacional (experiencia del equipo médico). Existen trabajos que demuestran que esta diferencia es especialmente significativa en los menores de 29 semanas.

Diversas experiencias en distintos países del mundo han demostrado que para mejorar estas cifras es importante organizar el cuidado neonatal de modo que se pueda entregar el mejor tratamiento y de la manera más oportuna, lo que es particularmente difícil en países de geografía como la nuestra. Para ello se han definido distintos niveles de cuidado perinatal, los que tratan RN de acuerdo al riesgo idealmente definido antes de nacer, concentrando los niños de mayor riesgo y aquellos que presentan patologías graves en grandes centros regionales. Gracias a estas estrategias se han conseguido importantes mejorías en la sobrevida de estos pacientes complejos.

Si bien la mayoría de los $\mathrm{RN}$ de alto riesgo que requieren cuidados especiales puede identificarse prenatalmente, aproximadamente un $25 \%$ de los niños que requieren cuidado intensivo no tiene factores de riesgo identificados antes de nacer. Es por esto que todos los niveles de atención perinatales deben estar capacitados para diagnosticar situaciones de riesgo y dar la asistencia necesaria para estabilizarlos. Junto con esto, disponer de un sistema de transporte bien equipado y con personal altamente capacitado para trasladar al RN que lo requiera en las mejores condiciones. Siempre que sea posible, debe preferirse el transporte prenatal de la madre y facilitar el nacimiento en un centro de mayor complejidad.

Existen otros aspectos de modernización que deben sumarse al desarrollo neonatal en forma prioritaria, siendo algunos de ellos el área informática, implementación de indicadores de gestión y calidad, favorecer la especialización y formación continua del recurso humano, promover la investigación y la humanización del trabajo en las áreas críticas y el desarrollo de procesos de información al usuario y familiares, 
consentimientos informados y orientación en relación a los problemas bioéticos del paciente crítico.

Un proceso de este tipo permite ir disminuyendo la variabilidad en los procesos clínicos, lo que a su vez favorece mejores evaluaciones de oportunidad, calidad e impacto en nuestras acciones para alcanzar el gran objetivo que nos convoca que es la mejor atención a nuestros pequeños pacientes.

En este organigrama asistencial es fundamental asegurar la formación continua de todos los profesionales de la salud que realizan la asistencia perinatal, establecer la comunicación y la coordinación continuada entre los diferentes servicios y disponer de un sistema común de control de calidad, que asegure la óptima utilización de los recursos y la igualdad de oportunidades.

En base a la información existente en la literatura y a la realidad nacional, la Directiva de la Rama de Neonatología de la Sociedad Chilena de Pediatría, con la colaboración de diferentes expertos de Centros Hospitalarios Nacionales, plantea las siguientes "Recomendaciones sobre Organización, Características y Funcionamiento de Servicios o Unidades de Neonatología" para que sirvan como orientación en la Gestión Neonatal.

\section{Consideraciones generales}

Existen revisiones de estándares neonatales en la literatura abarcando todas las áreas implicadas: niveles de atención, recursos humanos, equipamiento y estructura arquitectónica, entre otras.

Se considera necesario establecer una Política de Calidad con estándares mínimos que otorguen una adecuada atención al recién nacido y su familia con el objetivo de asegurar la mejor atención posible en cada unidad de acuerdo a su propia categoría y lograr una reducción de la morbimortalidad perinatal. Todo esto considera Gestión de Riesgos, Seguridad del paciente y Evaluación de Procesos y Resultados.

\section{Definición de los Servicios o Unidades}

Se define como Servicio o Unidad de Neonatología, la sección intrahospitalaria que ga- rantiza la cobertura asistencial de los pacientes neonatos, la asistencia y reanimación en la sala de partos o pabellón e incluye la atención del neonato que se encuentra en puerperio con su madre.

Desde el punto de vista operacional se considera período de atención neonatal desde el momento del nacimiento hasta las 44 semanas de edad post-concepcional, con un mínimo de 28 días y sin restricciones de peso al nacer.

Los pacientes hospitalizados mayores de 44 semanas de edad post-concepcional deben ser derivados a Servicios de Pediatría por haber superado el período de atención neonatal y la patología propia del neonato y corresponder a pacientes crónicos pediátricos.

\section{Criterios de admisión a Servicios o Unidades de Neonatología}

El Servicio o Unidad de Neonatología está constituido o conformado por la Unidad de $\mathrm{Pa}$ ciente Critico (UPC) y la Sección de Cuidados Básicos con el objeto de prestar cuidados en diferentes niveles de complejidad a neonatos con patología. La UPC se subdivide en $2 \mathrm{sec}-$ ciones, Unidad de Cuidado Intensivo (UCI) y la Unidad de Tratamiento Intermedio (UTI).

Un recién nacido puede transitar por estas distintas secciones del Servicio dependiendo de la dinámica de su patología, monitoreo y tratamiento.

Completan estas secciones, la Sala de Atención Inmediata u Observación, en donde se recibe al $\mathrm{RN}$ y la sección de Puerperio en donde está el RN sano con su madre.

Es importante destacar que la Sala de Atención Inmediata del Recién Nacido no es un lugar de hospitalización, sólo de transición por un período no mayor a 4 horas, por lo que un paciente que requiera observación más allá de ese tiempo, debe ser hospitalizado en un sector determinado del servicio, para allí seguir su estudio y manejo correspondiente. Excepcionalmente se manejará un paciente hospitalizado en Sala de Observación o Atención Inmediata si existe falta de disponibilidad transitoria de cupo para hospitalizado en la sección pertinente. 
La decisión última del lugar de ingreso al servicio es de responsabilidad médica. Los siguientes criterios definen el lugar correspondiente para hospitalizar a un RN en uno u otro sector:

\section{A. Unidad de Cuidados Intensivos (UCI)}

Es aquella dependencia de neonatología destinada a proporcionar cuidado médico y de enfermería permanente y oportuna a pacientes críticos inestables. Se caracteriza por contar con atención médica permanente, así como tecnología de alta complejidad.

Se denomina neonato crítico a aquel recién nacido enfermo cuya condición patológica afecta uno o más sistemas, que pone en serio riesgo actual o potencial su vida y que presenta condiciones de reversibilidad, que hacen necesaria la aplicación de técnicas de monitorización, vigilancia, manejo y soporte vital avanzado.

Criterios de Ingreso: Deben ser atendidos en este nivel de cuidado los pacientes que necesiten cualquiera de los siguientes manejos:

- RN con SDR agudo que requiere oxígenoterapia con $\mathrm{FiO}_{2} \geq 0,35$.

- $\mathrm{RN}$ que requiere cualquier tipo de apoyo ventilatorio, CPAP, Ventilación Mecánica Convencional, Sincronizada o VAF.

- RN con inestabilidad cardio-circulatoria y/o uso de drogas vasoactivas.

- RN con síndrome convulsivo en etapa aguda.

- RN con clínica de sepsis y/o meningitis bacteriana.

- $\mathrm{RN}$ en su post-operatorio hasta su estabilización.

- RN con apneas severas y repetidas de cualquier origen.

- $\mathrm{RN}$ con riesgo vital de cualquier otra etiología.

- RN con monitorización invasiva.

- $\mathrm{RN}$ prematuro < 1200 g hasta estabilización, definido como más de 5 días sin eventos de desestabilización.

- Peritoneodiálisis aguda.

- $\mathrm{RN}$ que requiere procedimiento invasivo.

\section{B. Unidad de Tratamiento Intermedio}

Es aquella dependencia de neonatología destinada al manejo de pacientes estables que requieren para su cuidado de monitoreo no invasivo, vigilancia y manejo de enfermería permanente además de cuidados médicos.

\section{Criterios de Ingreso}

Pacientes provenientes de la UCI y/o pacientes provenientes de cualquier otra sección que necesiten cualquiera de los siguientes manejos:

- SDR agudo con requerimientos de oxígenoterapia $\mathrm{FiO}_{2}<0,35$.

- Monitorización cardiorrespiratoria y/o saturación.

- Incubadora o cuna calefaccionada.

- Fleboclisis.

- Gastroclisis o alimentación por bomba a través de sonda enteral o gástrica.

- RN con alimentación parenteral.

- Cualquier tipo de catéter.

- $\mathrm{RN}$ con requerimientos especiales de enfermería.

\section{Unidad de Cuidados Básicos}

Es aquella dependencia destinada al manejo de pacientes totalmente estables sin riesgo conocido y que están hospitalizados por estudio o tratamiento de patologías no complejas, en espera de condiciones para el alta sin riesgo inminente y pacientes con fototerapia de bajo riesgo que no requieren para su cuidado de monitoreo no invasivo o vigilancia permanente. También se hospitalizará en esta sección los pacientes que no puedan ser trasladados junto a su madre en el período de post-parto.

\section{Criterios de Ingreso}

- RN que no cumpla ninguno de los requerimientos de los niveles atención en UCI ni Intermedio.

- RN prematuro en crecimiento, estabilizado en cuna y sin requerir monitoreo cardiorrespiratorio ni saturación permanente.

- RN de término en buenas condiciones generales; que no requiera monitorización cardiorrespiratoria ni saturación permanente.

- RN clínicamente estable con vía venosa permeable para tratamiento antibiótico.

- RN con requerimientos de enfermería habitual.

- $\mathrm{RN}$ en fototerapia sin riesgo conocido.

- $\mathrm{RN}$ con problema social o materno. 


\section{Criterios de egreso}

Los criterios de egreso de cada sección de hospitalizados corresponden al no cumplimiento de los criterios de ingreso correspondiente a esa sección y por tanto aumentar o disminuir la complejidad y corresponder a otra sección de hospitalizados.

\section{Criterios de alta}

Los criterios de alta del servicio corresponden a:

- Superar la condición o condiciones de patología que motivo el ingreso.

- Succión adecuada o cuando no es posible ésta lograr alimentación gastro-enteral expedita con padres o familiar responsable capacitado.

- Termorregulación.

- Padres o familiar o tutor responsable capacitado en la atención del neonato.

- Traslado a otra Unidad o Servicio dentro o fuera del hospital.

\section{Clasificación de los Servicios o Unidades Neonatales}

Se definirán de acuerdo a número de nacimientos, equipamiento (capacidad resolutiva) y posibilidad de traslado a unidad de mayor complejidad (distancia y accesibilidad).

\section{Unidades de Nivel I: Definidas como Unidades de Cuidados Básicos}

Ubicadas en Hospitales Tipo 3 y 4 de ciudades pequeñas, dentro de un Servicio de Pediatría General y con maternidades que atienden menos de 1000 partos al año.

\section{Características de la Unidad Neonatal Nivel I}

- Dar atención a RN de término sin patologías previstas.

- Otorgar cuidados básicos y tratamientos de patologías simples como: problemas de termorregulación, manejo de fototerapia y glicemias límites.

- Disponer de reanimación en sala de partos o pabellón, con personal entrenado en reanimación cardiopulmonar neonatal de acuerdo a estándares internacionalmente acepta- dos, como los de la Academia Americana de Pediatría y Academia Americana del Corazón.

- Estar capacitados para estabilización de los recién nacidos que requieran ser trasladados.

- Tener capacidad de trasladar a centros de referencia de mayor complejidad, bajo criterios de derivación previamente establecidos.

En la práctica todo RN patológico debiera trasladarse. En la situación actual las Unidades Neonatales Nivel I deben ser consideradas una Sección en Servicios de Pediatría General y en un futuro próximo debieran tender a desaparecer este tipo de unidades.

\section{Requerimientos}

- 2-3 cupos por cada 1000 nacidos vivos, dependiendo de la distancia y acceso geográfico a centro de derivación

- Disponibilidad de un médico que pueda acudir en pocos minutos, entrenado en reanimación neonatal y capaz de reconocer aquellos $\mathrm{RN}$ que requieran derivación, y enfermera o matrona entrenada disponible en el sitio.

- Equipamiento de reanimación y estabilización del recién nacido enfermo hasta su traslado: cuna radiante o incubadora, bolsa de ventilación a presión positiva, laringoscopio, oxígeno, aspiración, monitor cardiorrespiratorio, monitor de saturación, bombas de infusión continua, equipo de fleboclisis y soluciones endovenosas.

- Requiere sistema de traslado/referencia organizado a unidades nivel II o III. Incubadora de transporte y personal adiestrado en traslados realizado por centro receptor.

\section{Situación especial por distancia $o$ aislamiento}

En caso de que el establecimiento se encuentre a una distancia de más de $100 \mathrm{~km} \mathrm{o}$ tiempo de traslado de 2 horas o más de transporte terrestre a centro de mayor complejidad, se deberá disponer de medios para poder ofrecer, además de lo descrito, lo siguiente:

- Atender a RN que requieran cuidados básicos y tratamientos de patologías poco complejas (niños con dificultad respiratoria moderada con requerimientos de $\mathrm{FiO}_{2}<30 \%$, 
tratamientos ev, antibióticos por sospecha de infección con pacientes estables, hipoglicemias, hiperbilirrubinemia)

- Capacidad de dar reanimación avanzada y estabilizar a todos los RN nacidos en el centro

- Capacidad para estabilizar un prematuro o RN enfermo previo a su traslado.

En estos casos, estos hospitales deberán contar con equipamiento y recursos adicionales a los descritos para los centros tipo I.

\section{Requerimientos Unidades Nivel I}

con accesibilidad compleja:

- 4-5 cupos por cada 1000 nacidos vivos.

- Disponer de 1-2 incubadoras.

- Requiere de pediatra disponible y matrona o enfermera capacitada en atención neonatal con funciones específicas en la Unidad.

- Requiere equipamiento para montar transitoriamente al menos un cupo de UCI y realizar reanimación avanzada (cuna radiante, bolsa de resucitación manual, red de gases, monitores, bombas de infusión continua, y un ventilador mecánico para la estabilización/reanimación del paciente).

- Requiere sistema de traslado/referencia organizado a una unidad de mayor complejidad a cargo de un centro receptor.

\section{Unidades de Nivel II: Unidad de Cuidado Intermedio}

Este nivel de resolución debe estar inserto dentro de un hospital general (2 y 3) e idealmente con un Servicio de Pediatría que desarrolle algunas de las áreas específicas pediátricas de atención al niño

- Atención a RN $>34$ semanas y/o $>2000 \mathrm{~g}$.

- Disponible en maternidades que atienden un número de partos entre 1000 a $3000 \mathrm{al}$ año. Número mayor de partos requiere implementar el nivel superior de resolución.

- Capacidad de recepción de pacientes derivados de Unidades Neonatales Nivel I.

- Estabilizar un RN Pret $<2000$ gramos o $<34$ sem para su traslado a centros de mayor complejidad.

- Reingreso de recién nacidos procedentes de unidades de Nivel III que se encuentren en etapa estable (transporte de retorno).
- Otorgar cuidados de oxígenoterapia y presión positiva continua en vía aérea (CPAP) nasal.

- Practicar ventilación mecánica convencional de RN mientras se estabiliza y se espera su traslado a una Unidad Nivel III.

- Practicar exsanguineotransfusión.

\section{Requerimientos}

- La Unidad de Nivel II dispondrá de dos áreas de atención claramente definidas:

- Cuidados intermedios/básicos: 5-6 cupos por cada 1000 nacidos vivos.

- Cuidados de estabilización: 1 cupos por cada 1000 nacidos vivos.

- Requiere equipamiento necesario para dar cuidado para estabilizar a $\mathrm{RN}$ que requiera Cuidado Intensivo.

- Requiere tener un sistema organizado de transporte/referencia de pacientes complejos a una Unidad de Nivel III.

\section{Unidades de Nivel III: Unidades de Cuidado Intensivo}

Deben cumplir con las siguientes características:

- Estar integradas dentro de un hospital de referencia (Tipo 1 y 2) o regional, con maternidad e idealmente con un Servicio de Pediatría donde se desarrollen la mayoría de las sub-especialidades pediátricas o en su defecto disponer de interconsultores de las subespecialidades pediátricas más habituales (cardiología, neurología, broncopulmonar, cirugía infantil, gastroenterología, endocrinología, infectología, entre otras) que asis$\tan$ a los neonatos que lo requieran idealmente en el misma unidad de neonatología. Sólo se deben trasladar pacientes para evaluación de especialistas en áreas muy específicas (neurocirugía, cardiocirugía, cirugía oftalmológica, exámenes especiales).

- Junto a maternidades que atienden a más de 3000 partos anuales.

- Disponibilidad a ser centros de referencia de Unidades nivel II.

Las Unidades de Nivel III se clasifican en 2 sub-niveles:

Unidades Nivel III A. Además de la atención nivel II, incluye: 
- $\mathrm{RN}$ con edad gestacional superior a 27 semanas.

- Practicar apoyo ventilatorio con disponibilidad de ventiladores mecánicos sincronizados.

- Recién nacidos con falla multiorgánica o soporte multisistémico.

- Realizar procedimientos complejos como drenaje pleural, exsanguíneotransfusión, diálisis peritoneal, cateterismo umbilical y/o percutáneo.

- Nutrición parenteral.

- Capacidad de realizar intervenciones de cirugía neonatal.

\section{Requerimientos}

La Unidad de Nivel III A dispondrán de tres áreas:

- Área de cuidados intensivos (UCI): 1 cupo por cada 1000 nacidos vivos en el área de referencia.

- Área de tratamiento intermedio: 3-4 cupos de intermedio por cada 1000 nacidos vivos.

- Área de cuidados básicos: 1-2 cupos por cada 1000 nacidos vivos.

- Disponibilidad de técnicas de imagen: radiología portátil, ecografía doppler cerebral y abdominal portátil, ecocardiografía portátil, acceso a TAC.

- Disponibilidad de cirugía general pediátrica.

- Equipamiento necesario para dar cuidado intensivo.

- Disponibilidad de sub-especialidades pediátricas (cardiología, neurología, broncopulmonar, infectología, gastroenterología, etc) y especialidades de apoyo (otorrinolaringología, dermatología, oftalmología, etc).

- Sistema organizado de transporte y referencia de RN complejos desde y hacia Unidad Nivel II.

- Sistema de seguimiento de los niños de riesgo dado de alta.

\section{Unidades Nivel III B}

Corresponde a unidades con resolución de problemas específicos, de manejo supraregional y/o Nacional.

Deben estar presentes en hospitales con Centros de Alto Riesgo Obstétrico, con área de referencia mayor a 10000 nacidos vivos y distribuidas estratégicamente por distancia o complejidad.
Además de la atención propia del Nivel III A se tratarán en estos centros:

- Atención a todo tipo de recién nacidos tanto médicos como quirúrgicos, de cualquier peso y edad gestacional, dentro de los límites de la viabilidad.

- $\mathrm{RN}$ con HPP grave que requieran ventilación de alta frecuencia y administración de NO inhalado.

- Cuidado de RN con problemas quirúrgicos y no quirúrgicos que no son posibles de resolver en Nivel III A.

- Deben disponer de una unidad completa de rescate con equipamiento, movilización y personal entrenado.

- Deben atender a todos los RN $\leq 27$ semanas EG.

\section{Requerimientos}

- La Unidad de nivel III B con atención de patologías específicas dispondrán de tres áreas:

- Área de cuidados intensivos: 1,5 a 2 cupos por cada 1000 nacidos vivos con un mínimo de 6 cupos.

- Área de cuidados intermedios: 6 cupos por cada 1000 nacidos vivos.

- Área de cuidados básicos: 2-3 cupos de por cada 1000 nacidos vivos.

- Disponibilidad de técnicas de imagen avanzadas: ecocardiografía, TAC cerebral o RNM, medicina nuclear, radiología portátil, ecografía doppler portátil.

- Disponibilidad de sub-especialidades pediátricas (cardiología, neurología, broncopulmonar, infectología, gastroenterología, etc) y especialidades de apoyo (otorrinolaringología, dermatología, oftalmología, etc).

- Disponibilidad de cirugía pediátrica para realizar cirugía mayor en el hospital y subespecialistas para cirugías específicas (Neurocirugía, Urología Infantil, Cirugía Oftalmológica, Cirugía vascular, etc).

- Requiere equipamiento necesario para tratamiento intensivo complejo: ventiladores mecánicos sincronizados, monitores, VAF, disponibilidad de óxido nítrico.

- Sistema de seguimiento de los pacientes de riesgo dados de alta. 
- Requiere tener un sistema organizado de transporte/referencia de pacientes complejos con riesgo vital desde servicio o unidades de menor complejidad y para ECMO, cirugía cardiovascular, etc, y sistema de transporte de retorno.

\section{Requerimientos especiales a la realidad neonatal}

- Para lograr una real regionalización en salud y uso racional de equipos, infraestructura y recurso humano es absolutamente prioritario que exista un sistema expedito de rescate neonatal por región, dependiente de la unidad de mayor complejidad establecida en ella.

- Este sistema de rescate debe disponer de movilización adecuada a las condiciones de distancia, climáticas y de gravedad del neonato y que considere: Incubadora con monitores, bombas de infusión en número suficiente a la gravedad del paciente, ventilador mecánico, todos los equipos con adecuada autonomía y personal médico, enfermera o matrona y técnico paramédicos, todos con capacitación nivel intensivo neonatal y con disponibilidad 24 horas al día.

- Los hospitales en ciudades alejadas de centros de derivación por razones geográficas, deberán tener implementación que permita resolver localmente la gran mayoría de las patologías de riesgo vital, independiente del número de partos por año, previo al traslado si correspondiese.

- Además de lo anterior, se debe disponer de unidades o convenios con centros que resuelvan patologías cardiacas que requieran cirugía con circulación extracorpórea y posibilidad de realizar oxigenación por membrana extracorpórea (ECMO) para pacientes con falla respiratoria severa reversible.

- Es necesario y prioritario establecer a nivel ministerial una central de coordinación de camas criticas neonatales.

- Es necesario realizar seguimiento especializado, integral y con atención preferente a pacientes que recibieron terapia compleja.

\section{Unidades de Apoyo}

Se requiere acceso expedito durante las 24 horas a unidades de apoyo:
- Radiología. Ecografía.

- Scanner.

- Laboratorio.

- Hematología.

- Bioquímica.

- Banco de Sangre.

- Farmacia.

- Esterilización.

- Ambulancia.

- Servicios Generales.

- SOME o SAP (Servicio Administrativo).

- SEDILE.

\section{Requisitos técnicos sanitarios}

El nivel superior debe asumir todos los requisitos técnicos sanitarios de los niveles inferiores. Los hospitales con Unidades Nivel III deben coordinarse con sus centros de referencia Nivel I y II para:

1. Asegurar la recepción de todos los pacientes que lo requieran y le correspondan o en su defecto disponer de red paralela que pueda resolver el problema.

2. Disponer de sistema de transporte de rescate de los pacientes y del transporte de retorno, una vez resuelto el problema que motivó el ingreso.

3. Exigencia de formación continúa para el personal del propio hospital y de los centros de referencia.

4. Promoción de la investigación, con asignación de horas para este fin.

\section{Recursos humanos}

Recursos profesional médico: Profesionales médicos neonatólogos con especialidad acreditada según Reglamento de Certificación de las Especialidades y Subespecialidades de los prestadores individuales de salud, Decreto Supremo N 57/2007.

\section{Medico Jefe de Neonatología}

La Unidad o Servicio de Neonatología estará a cargo de un médico jefe, con jornada de trabajo de 33 hrs semanales, quien será responsable de las funciones de planificación y coordinación del trabajo interno, incluido el personal 
profesional y técnico de atención inmediata además de la coordinación con servicios clínicos y unidades de apoyo del hospital. En centros con más de 50 cupos totales debe existir una subJefatura con horas asignadas para ese fin.

Velará por el mejoramiento continuo de los procesos de la unidad, identificando los problemas y proponiendo las soluciones acorde a ellos.

Procurará disponer de un adecuado sistema de registros e indicadores que den cuenta de la gestión de la unidad.

A su cargo se encuentran todos los profesionales médicos y la matrona o enfermera jefe o coordinadora de la unidad y a través de ella todas las matronas o enfermeras del servicio y funcionarios no profesionales. Dado la alta complejidad de los servicios o unidades de neonatología es fundamental especificar estas dependencias en el organigrama.

\section{Recursos médicos de tratancia}

Proporcionarán atención directa a los pacientes asignados. Coordinarán el trabajo entre el equipo de salud de neonatología, de tal modo que la prestación médica sea un todo integrado que favorezca al paciente y su familia desde el punto de vista físico, psicológico y social.

Informarán en forma clara y veraz acerca del estado clínico de los pacientes a sus familiares o responsables directos.

Los recursos se deben distribuir de la siguiente manera:

- 22 hrs semanales por cada 3 cupos de cuidado intensivo.

- 22 hrs semanales por cada 5-6 cupos de tratamiento intermedios.

- 22 hrs semanales por cada 6-7 cupos de cuidados básicos.

Estos tiempos consideran asistencialidad, visita clínica, participación en entrega de turno, manejo integral de la ficha clínica, ingreso de información clínica a registro computacional local, auditoria, información expedita a los padres y capacitación interna con reunión semanal.

\section{Recurso médico en puerperio}

Realizarán atención matinal en la sección de puerperio en recién nacidos sanos o con patología que no requiere manejo hospitalizado pre- ocupados de la pesquisa de problemas del neonato o del binomio madre e hijo. Deben establecer políticas de apoyo a lactancia materna y educación en prevención.

- Se requiere $22 \mathrm{hrs}$ médicas semanales por cada 24 camas en puerperio; $6 \mathrm{RN}$ por hora que permita cumplir con objetivos de apoyo lactancia, educación, prevención, evaluación médica de todos los RN de alta y los neonatos o binomios con evolución no fisiológica o con patología.

- Refuerzo médico matinal los fines de semana y festivos.

\section{Recursos médicos de residencia}

Realizarán turnos para dar cobertura permanente de atención. Dado que en los servicios o unidades de neonatología su ámbito de acción incluye diferentes actividades la recomendación debe considerar a lo menos número de partos, cupos totales y número de cupos de Intensivo. Considerando lo anterior la recomendación es la siguiente:

- 1 cargo de médico residente cuando existan cupos de UCI, independiente del número de partos y/o capacidad total de la unidad.

- 2 cargos de médico residente si existen 2 de las siguientes condiciones:

- Servicio o unidades con más de 5000 partos al año.

- Capacidad total de neonatología mayor o igual a 25 cupos.

- Capacidad de UCI de 5 a 10 cupos.

- Responsabilidad de transporte de paciente grave de ida o rescate desde y hacia otras unidades.

- 3 cargos de médico residente si existen al menos 3 de las siguientes:

- Servicio o unidades con más de 8000 partos al año.

- Capacidad de UCI mayor a 10 cupos.

- Capacidad total de la unidad o servicio mayor a 50 cupos.

- Responsabilidad de transporte de rescate desde unidades de menor complejidad asignadas por red.

\section{Recursos profesional no médico Matronas o Enfermeras}

El equipo estará compuesto por matronas o 
enfermeras diurnas y matronas o enfermeras clínicas en turno.

\section{Matrona o Enfermera Jefe o Coordinadora}

Requiere asignación de jornada de trabajo de 44 hrs semanales en horario diurno y cumplirá funciones administrativas, colaborará en la función clínica y velará por la continuidad de la atención integral del paciente. Su dependencia técnica y administrativa es del Médico Jefe de Neonatología.

\section{Matrona o enfermera diurna}

En unidades o servicio con más de 15 cupos se requiere matrona o enfermera diurna con asignación de horario diurno completo y cumplirá funciones a cargo de insumos, equipos médicos, apoyo a estación clínica, entre otras funciones.

En unidades mayores a 40 cupos se requieren 2 matronas o enfermeras para esta función y en caso de más de 60 cupos se requieren 3 matronas o enfermeras diurnas.

\section{Matronas o enfermeras clínicas}

Las matronas o enfermeras clínicas de neonatología son responsables de la atención directa del paciente, correspondiéndoles formular y ejecutar tareas especificas según las condiciones del paciente, ejecutar las indicaciones médicas, supervisar el cumplimiento del plan de atención de enfermería y llevar todos los registros de los pacientes a su cargo. Estas se dividen según el área de su quehacer.

\section{Matrona o enfermera clínica Cuidados Intensivos}

La dotación de matronas o enfermeras clínicas por turno será de 1 por cada 3 cupos. Debe existir un sistema de refuerzo de matronas o enfermeras de llamada en caso de neonatos extremadamente críticos que requieren un profesional de enfermería exclusivo.

\section{Matrona o enfermera clínica Tratamiento Intermedios}

La dotación de enfermeras clínicas por turno será de 1 por cada 6-7 camas.

Matrona o enfermera de Cuidados Básicos

La dotación de matrona o enfermeras clínicas en Cuidados básicos será de 1 por cada 10-
12 camas, en horario diurno de 12 horas todos los días del año.

\section{Matrona en Sala de Observación}

Las matronas de neonatología en Sala de Observación son responsables de la atención neonatal inmediata directa al momento del parto, correspondiéndoles realizar la atención inmediata y deben estar capacitadas en reanimación neonatal. Además deben tener capacidad para pesquisar neonatos de riesgo para solicitar pronta atención por médico e implementar medidas inmediatas para favorecer la estabilización. Deben llevar todos los registros de los pacientes nacidos. La dependencia técnica y administrativa corresponde al Servicio o Unidad de Neonatología.

La dotación será de 1 por cada 4 000-5 000 partos en sistema de turno permanente.

\section{Recurso kinesiólogo en neonatología}

Dada la alta complejidad de los pacientes de unidades neonatales y su necesidad de atención kinésica integral tanto respiratoria como de neurorehabilitación y considerando el amplio uso de ventilación mecánica, retraso neuromotor y los altos costos en salud implicados, es indispensable contar con cobertura de kinesiólogo diurno en las Unidades de Neonatología Nivel III. En unidades con menos de 40 cupos totales 22-33 hrs de kinesiología y en las unidades con más de 40 cupos se requieren 44 hrs semanales de kinesiólogo.

Además se deben definir estrategias de atención kinésica respiratoria de modo de asegurar cobertura nocturna, aprovechando las necesidades de todo el hospital y ampliándolas de modo de asegurar cobertura de atención a pacientes en otros servicios del hospital. Estos profesionales deben poseer un elevado nivel técnico profesional, deben demostrar conocimientos y experiencias para lo cual deben estar debidamente capacitados en rehabilitación neonatal respiratoria y motora.

\section{Otros Profesionales}

Se debe contemplar un expedito acceso a otro tipo de profesionales, como nutricionista, asistente social, psicólogos y médicos especialistas. 


\section{Recursos humanos no profesional universitario}

\section{Técnico Paramédico}

El técnico paramédico es el funcionario encargado de efectuar la atención de enfermería programada y asignada, en forma integral, oportuna, amable y humana. La dotación adecuada es de:

\section{Técnico Paramédico en Cuidados} Intensivos

La dotación por turno permanente será de 1 por cada 3 cupos.

\section{Técnico Paramédico en Cuidados Intermedios}

La dotación por turno permanente será de 1 por cada 4-5 cupos.

\section{Técnico Paramédico en Cuidados Básicos}

1 técnico paramédico por cada 6 camas en rotativa de turnos permanente.

\section{Técnico Paramédico diurno}

El técnico paramédico diurno es el funcionario encargado de apoyo en la atención de enfermería programada y asignada, además de funciones en preparación de fármacos en estaciones de enfermería y apoyo en procedimientos médicos y de enfermería. Un técnico por cada 20-25 cupos totales.

\section{Técnico paramédico en Sala}

\section{de Observación}

La dotación será de 1 por cada 2 000-2 500 partos en sistema de turno permanente. Si la política del servicio es realizar apego de 30 minutos debe aumentarse la dotación en un $50 \%$.

\section{Auxiliar de Servicio}

Es el personal encargado de efectuar y mantener el orden, limpieza y desinfección del material, equipos y planta física.

Colabora con el técnico o auxiliar de enfermería en labores de confort, control de ropa, traslado de exámenes y realiza además funciones de mensajero.

La dotación debe ser de a lo menos 1 auxi- liar de servicio por cada 12 camas, en turnos que cubran las 24 horas.

\section{Personal a cargo de Recepción}

Es el personal con funciones de orientación, educación y control de ingreso de padres.

La dotación es de una auxiliar u orientadora todos los días del año en sistema de turno díadía-libre-libre en el horario destinado a visitas.

\section{Secretaria}

Es el personal encargado de funciones de secretaria y administrativas del Servicio. En los Servicios Nivel III la jornada laboral debe ser de 44 horas semanales. En Servicio con más de 45 cupos se requieren 2 secretarias.

\section{Equipamiento e infraestructura}

\section{Equipamiento}

El servicio o unidad de neonatología deberá estar dotada del equipamiento necesario para otorgar un adecuado soporte vital del paciente neonato enfermo, recuperar su gravedad hasta lograr condición de estabilidad y mejoría, además poder dar el apoyo necesario de confort cuando no podamos curar.

Se deben ir incorporando todos los avances tecnológicos que se requieran de acuerdo al nivel de complejidad correspondiente.

Deberá contar con el siguiente equipamiento:

\section{General}

- Carro de paro completo.

- Monitor desfibrilador con placas neonatales en unidades Nivel II y III.

- Fonendoscopio 1 por cada cupo.

- Laringoscopio 1 por cada 5-6 cupos de intermedio con hojas $N^{\circ} 00-0-1$.

- 1 equipo de rayos portátil.

- 1 ecógrafo-ecocardiógrafo con Doppler color en unidades Nivel III.

- Electrocardiógrafo con placas neonatales y precordiales sin succión.

- Incubadora de transporte con ventilador de transporte, 2 bombas de infusión y monitor con ECG y saturometría, todos con autonomía mínima de 2 hrs en centro de Nivel II y III. Este requerimiento se puede eliminar en 
centros Nivel II si existe adecuado y asegurado Sistema de Rescate desde Nivel III.

- Incubadora de transporte básica para traslados locales o de baja complejidad. Siempre disponible en toda unidad neonatal independiente de la complejidad. Una incubadora simple en unidades con menos de 30 cupos, 2 unidades en servicios de 30-45 cupos y 3 en servicios con más de 45 cupos totales.

- Carro multiuso de material limpiable no poroso, una cada 3 cupos en intensivo, una cada 4-6 cupos en intermedio o básico.

- Portasueros. Tres portasueros de base amplia por cada cupo de UCI, 1 portasuero estándar por cada cupo de intermedio.

- Calentador de mamaderas termorregulado. Un equipo cada 20 cupos de hospitalización. Siempre debe existir un equipo de reposición.

- Refrigerador para medicamentos.

- Oto-oftalmoscopio, 1 por unidad.

- Balanzas digital gramo a gramo 1 por cada 10 cupos de hospitalizados.

- Negatoscopio.

- Reloj mural, 1 cada 6 cupos.

- Termómetro ambiental uno por cada 12-15 metros cuadrados de sala de atención.

- Equipo de emisiones otoacústicas.

\section{Cupo Intensivo}

- Cuna calefaccionada servocontrolada en 30$40 \%$ de los cupos.

- Incubadora de intensivo doble pared con servohumedad y pesa digital incorporada en $70 \%$ de los cupos.

- Monitor multiparámetros con ECG, saturometría, frecuencia respiratoria y apnea, presión arterial no invasiva, 2 canales para presión invasiva, temperatura 1 por cada cupo.

- Adicionar modulo EEG cada 3-4 cupos de UCI.

- Ventilador sincronizado con pantalla de curvas, 1 por cada cupo.

- Ventilador de alta frecuencia y óxido nítrico inhalado cada 5-6 cupos de UCI en unidades Nivel III B.

- 4-5 bombas de infusión de jeringa por cada cupo.

- 4-5 bombas de infusión peristálticas o volu- métricas por cada cupo.

- Equipos de fototerapia 1 cada 2 cupos.

- Balanza digital 1 cada 3-4 cupos.

- Mezclador aire-oxígeno 1 cada 3 cupos.

- Base calefactores para oxígenoterapia por halo, 1 cada 3 cupos.

- Halo o Hood 1 cada 3 cupos.

- Oxímetro ambiental 1 cada 3 cupos.

- Lámpara de procedimiento 1 cada 3-4 cupos.

- 1 resucitador manual por cada cupo.

- Laringoscopio 1 por cada 3 cupos con hojas $\mathrm{N}^{\mathrm{o}} 00-0-1$.

\section{Cupo Intermedio}

- Cuna calefaccionada en 10\% de los cupos.

- Incubadora doble pared en $70 \%$ de los cupos.

- Cuna corriente con cajones en $20 \%$ de los cupos.

- Monitor multiparámetros en 50\% de los cupos, con ECG, saturometría, frecuencia respiratoria y apnea, presión arterial no invasiva, temperatura

- Monitor de saturación en 50\% de los cupos, idealmente con PANI incluida.

- 1 bomba de infusión por jeringa por cupo.

- 1 bomba de infusión peristáltica por cupo.

- Equipos de fototerapia 1 cada 3 cupos.

- Balanza digital 1 cada 5-6 cupos.

- Mezclador aire-oxígeno 1 cada 3 cupos.

- Base calefactores para oxígenoterapia por halo, 1 cada 3 cupos.

- Halo o Hood 1 cada 3 cupos.

- Flujometro de litro 1 por cada 3-4 cupos.

- Oxímetro ambiental 1 cada 3-4 cupos.

- Monitor de presión arterial 1 cada 3-4 cupos.

- Lámpara de procedimiento 1 cada 5-6 cupos.

- 1 resucitador manual cada 5-6 cupos.

- Laringoscopio 1 por cada 5-6 cupos con hojas $\mathrm{N}^{\mathrm{0}} 00-0-1$.

- 1 podómetro por cada 8-10 cupos.

- Microflujometros de oxígeno en 30\% de los cupos.

\section{Cupo básico}

- Cuna corriente con cajones en 100\% de los cupos.

- Monitor de saturación en 40\% de los cupos.

- 1 bombas de infusión por jeringa por cada 6 cupos. 
- Equipos de fototerapia 1 cada 3 cupos.

- Balanza digital 1 cada 6 cupos.

- Monitor de presión arterial 1 cada 6-8 cupos.

- Colchón de agua o aire termorregulado 1 cada 4-5 cupos.

- 1 podómetro por cada 8-10 cupos.

- Microflujometros de oxígeno en $40 \%$ de los cupos.

Cupo en Sala de Atención Inmediata u Observación

- Cuna calefaccionada 1 cada 1 000-1 500 partos.

- Cuna corriente que permita traslado 1 cada 1 500-2 000 partos.

- Calefactor radiante con servocontrol 1 por cada 4 cupos.

- Laringoscopio con hojas $\mathrm{N}^{0} 00-0-1,1$ cada cuna calefaccionada.

- Balanza digital 1 por sala.

- Podómetro 1 cada 3-4 cupos.

- Monitor de saturación 1 por sala. En sala con más de 4 cunas calefaccionadas se requieren 2 monitores.

- Monitor de presión arterial 1 por sala.

- Resucitador manual 1 en cada cuna calefaccionada.

- Refrigerador de vacunas, el cual también puede estar en puerperio.

- Reloj digital en cada cupo de reanimación.

Sería recomendable disponer de reanimador neonatal de ventilación controlada, con control de tiempo y presión de vía aérea constante con el fin de reanimar con parámetros conocidos.

En caso de atención neonatal en el pabellón de parto debe disponerse de equipamiento completo e individual en cada pabellón.

\section{Lactario}

Además el Servicio o Unidad de Neonatología debe disponer de Lactario implementado con:

- Sala con adecuada ventilación con mínimo 2 metros cuadrado por cada unidad de lactario con condición de privacidad para la madre.

- Ubicado con acceso inmediato a salas de hospitalizados.

- Bombas de extracción de leche 1 por cada 3-4 cupos de hospitalización.

- Casillero individual.
- Lavatorio accionado por pedal, codo o sensor de movimiento con área limpia y área sucia.

- Mesón de superficie lavable.

- Mueble con cubierta lavable para almacenamiento de insumos.

- Refrigerador exclusivo para mamaderas.

- Personal profesional no médico a cargo en días hábiles.

- Auxiliar o técnico en horario diurno todos los días de la semana.

\section{Planta física}

Es evidente que con la aparición del concepto del "cuidado centrado en la familia" los espacios tienen que incrementarse. Actualmente, la tendencia es a que la familia permanezca el mayor tiempo posible cerca del recién nacido hospitalizado, sin limitación de horarios, por lo que los espacios originalmente dedicados a los cuidados básicos y especiales sean ahora muy insuficientes y se requiere disponer de al menos $7 \mathrm{~m}^{2}$ en promedio por paciente para garantizar este fin, además de todas los necesidades asociadas a la prolongada presencia de los padres y familia en los hospitales, por lo tanto se deben considerar espacios de estar, lugares para alimentarse y adecuados servicios higiénicos.

Una situación que se debe tener muy cuenta es que el plan de construcción o reforma que planteamos no debe ser rígido en ningún aspecto, pues "solamente unas cuantas normas y pautas han soportado el paso del tiempo, y no hay que engañarse al pensar que el plan escogido será siempre perfecto y vigente". Ejemplo de ello es lo indicado en las "Guías de Atención Humanizada del proceso Reproductivo" que nos da pauta referido a los nuevos espacios y requerimientos en la atención neonatal.

Las unidades neonatales históricamente han sido pequeñas, con espacios insuficientes e inadecuados para almacenamiento y necesidades del personal y las familias.

1. Configuración general: El Servicio o Unidad de Neonatología debe tener una configuración tal que permita cumplir con los objetivos y metas para la cual ha sido creada para el nivel correspondiente de complejidad. Debe 
cumplir con los mínimos requeridos para equipamiento y disminuir riesgo de infecciones nosocomiales, accidentes y permitir el expedito flujo y estancia de pacientes, equipo de salud y padres. Debe disponerse de espacio para asistencia, bodegaje, equipos, capacitación y espacios administrativos y de gestión.

2. Localización dentro del hospital: Debe estar cerca de las salas de parto y pabellones de maternidad y lejos del transito de personas del hospital. Para las unidades que reciben traslados debe asegurarse un acceso expedito desde donde llegan las ambulancias al hospital.

\section{Areas mínimas, espacios requeridos:} El espacio para cada recién nacido en UCI debe ser de un mínimo de 9 a $11 \mathrm{mts}^{2}$, excluyendo lavamanos y pasillos. Debe existir, además, un pasillo adyacente a cada cupo de UCI que permita el expedito flujo de equipos.

Debe asegurarse un espacio suficiente para permitir la privacidad del paciente y su familia, para lo cual también se debe minimizar la circulación de personas entre cunas y/o incubadoras.

En cuidado intermedio la superficie mínima por cupo será de 4 a $5 \mathrm{~m}^{2}$, además del espacio para la circulación general ya descrito. La distancia entre cunas debe ser como mínimo de 1 metro por lado, $1,2 \mathrm{~m}$ en incubadoras de nivel intermedio y esta distancia aumenta a $1,5 \mathrm{~m}$ por lado en caso de cupos de intensivo.

Las incubadoras y/o cunas calefaccionadas deben estar ubicadas en un espacio alejado de fuente de calor externa o exposición al sol que pueda alterar la temperatura o humedad entregada.

\section{4. Áreas para lavado de Incubadoras:} Debe existir un espacio físico para lavado de incubadoras, cunas calefaccionadas y limpieza de cunas corrientes con dos lavabos profundos, uno para sucio y otro para limpio. Las dimensiones deben permitir como mínimo el acceso expedito de 2 equipos en forma simultanea. En unidades con más de 40 cupos el tamaño de esta sala debe permitir acceso a 4 equipos. Este espacio debe disponer de estante para almacenar insumos de aseo de equipos.

\section{Sala de equipos: Debe tener capacidad}

para guardar a lo menos el $33 \%$ de los equipos o implementos propios de un servicio de neonatología.

6. Salidas eléctricas: Todas las salidas eléctricas deben permitir paso de corriente normal y de emergencia. Cada cupo de UCI debe disponer de 12 a 15 tomas de electricidad de 16A. En cupo de intermedio se debe contar con al menos 8 tomas de corriente. Todas las redes eléctricas que dan servicio a neonatología deben disponer de suministro complementario de energía eléctrica a través de grupo electrógeno como fuente propia de energía

7. Salidas de gases: Los gases medicinales deben contar con un sistema de vigilancia y control, debiendo existir una sectorización en las canalizaciones de gases por 2 ó 3 cupos de atención.

Cada salida de gases será un sistema individual permitiendo las reparaciones con la red en funcionamiento. En UCI debe haber acceso simultáneo de gases en cantidad de 2 para aire, 3 para oxígeno y 2 de aspiración; mientras que en intermedio deberán existir 1 para aire, 1 para oxígeno y 1 de aspiración. En cuidados básicos el $50 \%$ de los cupos deben disponer de salida de oxígeno y sistema de aspiración.

Cada toma de oxígeno debe tener su respectivo flujométro de 15 lts. En cuidado intermedio el $30 \%$ de ellos deben ser microflujometros (1,5 litros) y en cuidados básicos la totalidad deben ser microflujometros. Las tomas de vacío deben disponer de manómetros pediátricos en su sistema de regulación de presión.

8. Iluminación: La unidad deberá tener preferentemente iluminación natural, disponiendo el alumbrado artificial de varios tipos de iluminación y capacidad para atenuarse por la noche. Todos los puestos deben tener alumbrado individual. No se han establecido los niveles óptimos de iluminación. Se ha recomendado rangos ajustables de 10 a 600 lux (1 a 60 foot candles); existen estudios que demuestran beneficios al permitir las variaciones de la luz, bajando los niveles en la noche a 0,5 foot candles. En cada cupo debe haber luz en forma 
individual, que sea ajustable entre los niveles mínimos y máximos, minimizando las sombras y los reflejos. Estos cambios de luces no deben ser percibidos por los pacientes que están en cupos contiguos. Debe disponerse de al menos una fuente de luz natural en las áreas de cuidado del recién nacido. Las ventanas exteriores deben estar provistas de panel con doble vidrio o una cubierta aislante para minimizar las perdidas o ganancias de calor y deben estar equipadas para proveer sombra o ser opacas para evitar las distorsiones de la luz.

9. Superficie del suelo: Debe ser fácil de limpiar para evitar el crecimiento bacteriano y resistir el uso de químicos. El material para la superficie del suelo debe ser durable (zona de alto trafico) y tener propiedades acústicas. $\mathrm{La}$ densidad del material usado debe resguardar del ruido y ser confortable. Debe tener una resina protectora, impermeable y las uniones del piso deben tener sellos químicos y propiedades antimicrobianas y antiestáticas.

10. Superficie de las paredes: Deben ser fácil de limpiar, durables y con propiedades acústicas. Los materiales a considerar son: pinturas lavables, cubiertas de vinílico con absorbente de sonido. Las ventanas y muros no deben generar condensación.

11. Techos: No deben acumular polvo, la superficie debe ser lisa, totalmente lavable y tener sistema acústico.

12. Áreas para lavamanos: En cada pieza individual debe haber un lavamanos. En las áreas con múltiples cunas o incubadoras deben existir uno cada 6 cupos y el desplazamiento entre la atención del paciente y el lavamanos debe ser como máximo de 6-8 metros.

El lavamanos debe tener un tamaño suficiente como para evitar el salpicado y la retención de agua. Las dimensiones mínimas recomendadas son $60 \mathrm{~cm}$. de ancho x $40 \mathrm{~cm}$ de adelante atrás x 25-30 cm de profundidad. Los lavamanos deben tener grifería tipo quirúrgica, vale decir llave accionable con el pie o codo o sensor de movimiento y cuello de cisne. Se debe asignar un espacio para dispensador de jabón, desinfectante, toallas desechables y basurero con pedal cubierto.

\section{Climatización: ambiente, temperatu-} ra, ventilación: El sistema de control de temperatura debe tener capacidad para mantenerse entre $24^{\circ}-28^{\circ} \mathrm{C}$ con una humedad relativa de 30 a $60 \%$, evitando la condensación en las paredes y ventanas. El equipo de climatización debe prestar servicio exclusivo a la unidad de neonatología y debe tener capacidad para funcionamiento permanente. Se requiere un mínimo de 10 a 12 movimientos de aire por hora con dos cambios de aire proveniente desde el exterior. El patrón de distribución de aire debe impedir el movimiento de material particulado en el espacio. Las entradas y salidas de aire deben estar ubicadas de manera de minimizar las corrientes de aire cerca de las cunas de los niños. El aire debe estar filtrado con un $90 \%$ de eficiencia. El sistema debe mantener el recinto en sobre presión, para evitar ingreso de aire desde otras áreas.

La toma de aire fresco del equipo de climatización debe estar situada por lo menos a más de 7,6 mt. de cualquier fuente contaminante tales como las chimeneas de los equipos de combustión, sistemas de aspiración, respiraderos de cañerías, etc.

14. Disminución del ruido: El área cerca del recién nacido no debe exceder a los 50 a 55 dB. Los sonidos transitorios no deben exceder a los $70 \mathrm{~dB}$.

15. Cubierta de los mesones, mesas de trabajo: Deben ser áreas fáciles de limpiar. Las superficies de los mesones deben tener el menor numero de uniones posibles, los bordes expuestos a impactos deben ser redondeados, el material de construcción no deber ser astillables o agrietable.

16. Pieza de aislamiento: En cada unidad de cuidado intensivo se debe asignar un espacio para recién nacidos que requieran aislamiento; junto a esta pieza debe existir un área para lavamanos, cambio de delantales y un lugar para guardar material limpio y sucio. El sistema de ventilación debe ser con aire a presión nega- 
tiva y el $100 \%$ del aire debe ser expulsado hacia fuera del recinto. Debe existir un sistema de comunicación de emergencia. La pieza de aislamiento debe tener un mínimo de $12 \mathrm{mts}$. cuadrados. Debe contar con ventana de observación y persianas para privacidad que sean fáciles de usar y limpiar. Debiera además contar con la posibilidad de monitorización a distancia.

17. Clínica de enfermería: Se debe sectorizar el área de trabajo clínico y el área de insumos y medicamentos. Debe contar con lavamanos con las características de grifería accionable con los codos, pies o sensor de movimiento y dispensadores de toalla, jabón y desinfectantes. Muebles de puerta corredera para guardar material, equipos, insumos y ropa. Los muebles y mesones clínicos deben ser de material lavable, anchos, color claro y lisos. A lo menos esta área debería tener una superficie mínima de 30 mts cuadrados.

18. Estación de Atención y monitoreo: Se debe asignar un espacio amplio de preferencia en el centro de la unidad de modo de facilitar la visibilidad y vigilancia de todos los puestos de atención. En servicios Nivel III se debe contar con central de monitorización.

19. Espacio para profesional médico: Espacio para trabajo de médicos, interconsultores y otros profesionales que brindan apoyo al trabajo como kinesiólogos, laboratorio, farmacia. Se debe contemplar un área para trabajo medico con escritorios y 1 terminal computacional conectado en red por cada 4-5 cupos de hospitalización.

20. Espacio para residencia: Se debe disponer de espacio de estar y residencia para profesional médico de turno, profesionales no médicos y personal no profesional universitario el cual debe disponer de espacio suficiente para descanso y baño privado con ducha. Estos espacios deben estar ubicados inmediatos al espacio clínico.

21. Area de recepción y atención de la familia del recién nacido: Se debe disponer de un área de recepción para la familia. En esta área se debe disponer de asientos, casilleros, lavamanos y personal que oriente en el ingreso. Se requiere disponer de sala para información a los padres y familia.

\section{Espacio para los padres y/o familia:} Los espacios para los padres pueden estar dentro de la unidad o inmediatamente adyacente a ella. Este espacio de estar para padres debe permitir tener privacidad, tener sillones confortables, casilleros, teléfonos, baños, acceso a material de educación que incluya revistas, métodos audiovisuales, información acerca de los cuidados que debe tener su recién nacido, etc.

23. Espacio para reuniones de los profesionales y funcionarios administrativos: Espacio para el trabajo clínico-administrativo en el interior de la unidad que permita el desplazamiento del personal con facilidad y que debe contar con lugares para el almacenamiento de fichas, documentos clínicos de uso permanente, conexión con la red informática del hospital, escritorios y sillas.

24. Auditórium o Sala de reunión: Se requiere disponer de sala de reunión multiuso que permita capacitación interna y docencia. El tamaño e implementación debe ser proporcional al número de funcionarios permitiendo la presencia simultánea de a lo menos el $33 \%$ de los funcionarios del servicio. Debe disponer de sillas, escritorio o mesa, pizarra, video-proyector, telón y todo el equipamiento propio de un auditórium.

25. Area de gestión administrativa: Considera oficina de Jefatura Médica y de Enfermería con área especifica y privada para gestión administrativa y oficina para gestión de secretaría, ambas con acceso telefónico y sistema computacional.

26. Espacio para bodega: Se requieren diferentes niveles de bodegaje, uno central de insumos de hospital y una segunda bodega adyacente al área de cuidado del recién nacido que permita guardar insumos usados frecuen- 
temente como: pañales, ropa blanca, delantales, papeles, medicamentos y fórmulas lácteas. El tamaño de esta área debiera ser $1 \mathrm{mt}^{2}$ por cada cupo de hospitalizado.

Debe existir un área sucia para guardar material usado y contaminado antes de ser removido. El sistema de ventilación de esta bodega, debe ser a presión negativa y el 100\% del aire expelido hacia afuera. Esta área sucia debe estar situada de manera que el retiro del material sucio no pase por el área de cuidado de los recién nacidos.

27. Seguridad: La unidad debe estar diseñada bajo un programa de seguridad para proteger la seguridad física de los recién nacidos, su familia y miembros del equipo de salud. Las necesidades de seguridad deben ser balanceadas con las necesidades de confort y privacidad de las familias.

\section{Generalidades:}

- La Unidad debe contar con redes informáticas suficientes y acorde con los rápidos avances de la informática en salud y el desarrollo de ficha clínica computacional, además de software de imagen y exámenes de laboratorio. Se debe disponer de 1 terminal conectado en red por cada 4 a 4,5 cupos de hospitalización.

- Deben existir al menos 2 líneas telefónicas con el exterior y comunicación interna con el resto de las unidades.

- Ante la necesidad de asegurar el funcionamiento permanente de las instalaciones de la unidad, se deberá contar con personal de mantenimiento las 24 horas del día.

\section{Mantención de equipos}

Debe existir programa de mantención preventivo según la recomendación del fabricante y hoja de vida de la totalidad de los equipos disponibles. Además contar con sistema expedito de reparación.

\section{Normas y manuales}

Con el fin de asegurar servicios de la mejor calidad, es imprescindible que los Servicios o Unidades de Neonatología se manejen por normas, guías y manuales que permitan uniformar criterios y estandarizar procedimientos, de manera de disminuir las variaciones. Estas normas deben estar actualizadas como máximo cada 23 años y deben estar en conocimiento y a disposición de todo el personal, y deben incluir, entre otras:

- Manual de Organización y Funciones.

- Guías de Atención Neonatal.

- Manual de Procedimientos de Enfermería.

- Normas de Infecciones Intrahospitalarias.

- Normas de Seguridad del Personal.

- Normas en caso de Desastre y Evacuación.

- Normas de Transfusión.

- Normas de Laboratorio referido a toma de muestra de exámenes.

- Normas de Farmacia referido a despacho de medicamentos y Nutrición Parenteral.

- Normas de Calidad.

\section{Sistema de registro y evaluación de calidad}

El sistema de registros dependerá de cada nivel local; en un futuro próximo debería crearse un sistema estándar para todo el país, con procedimientos computarizados.

Los registros deben ser documentos confidenciales, que pueden llegar a tener connotaciones legales. El lenguaje empleado debe ser técnico y detallado, con datos de máxima exactitud. En ellos no deben utilizarse abreviaturas que no sean de uso común.

Deberá desarrollarse un sistema de indicadores de cobertura y calidad de atención. Los registros deben permitir generar informes periódicos por grupos de peso y edad gestacional en los siguientes rangos:

- Por peso expresado en gramos al nacer: $<$ 500; 500-599; 600-699; 700-799; 800-899; 900-999; 1 000-1 249; 1 250-1 499; 1 5001749 ; 1 750-1 999; 2 000-2 499; 2 5002 999; 3 000-3 999; 4 000-4 499; >4 499 g.

- Por semanas cumplidas al nacer: $<24$, luego semana a semana desde las 24 semanas hasta las 37; 38-42 y > 42 sem. 
Estos informes deben incluir a lo menos:

\section{1) Informe General}

- $\mathrm{N}^{\mathrm{o}} \mathrm{RN}$ Hospitalizados, expresado en $\mathrm{N}^{\mathrm{o}}$ total y $\%$ en relación al grupo correspondiente de nacidos

- $\mathrm{N}^{\circ} \mathrm{RN}$ con Apgar 5min. $<\mathrm{o}=3$ expresado en $\mathrm{N}^{\mathrm{o}}$ total y tasa por mil.

- $\mathrm{N}^{\circ} \mathrm{RN}$ con Apgar 5 min. = 4-6 expresado en $\mathrm{N}^{0}$ total y tasa por mil.

- $\mathrm{N}^{\mathrm{o}} \mathrm{RN}$ fallecidos menores de 28 días, expresado en $\mathrm{N}^{\mathrm{o}}$ total y tasa por mil.

- $\mathrm{N}^{\circ}$ de $\mathrm{RN}$ fallecidos menores de 28 días con condición de mortineonatos incompatibles perse con la vida. Se expresa en $\mathrm{N}^{\mathrm{o}}$ por grupo y $\%$ de los mortineonatos de ese grupo.

- Tasa de mortalidad neonatal excluidos RN incompatibles perse. Esto corresponde a Tasa de Mortalidad Neonatal Corregida y se expresa por mil. Son los RN fallecidos menores de 28 días sin condición de incompatible perse con la vida.

- $\quad \mathrm{N}^{\mathrm{o}}$ Pacientes fallecidos mayores de 27 días, expresado en $\mathrm{N}^{\mathrm{o}}$ total y tasa por mil.

\section{2) Informe especifico}

- Tasa de Encefalopatía hipóxico isquémica (EHI), expresado en; $\mathrm{N}^{\mathrm{o}}$ total de $\mathrm{RN}$ con $\mathrm{EHI} / \mathrm{N}^{\mathrm{o}}$ nacidos vivos del período x $1000 \mathrm{y}$ $\mathrm{N}^{\mathrm{o}}$ de RN con EHI Grado II-III / $\mathrm{N}^{\mathrm{o}}$ nacidos vivos del período x 1000 .

- RN con infección intrahospitalaria referida a lo exigido por Comité de IIH, esto es Sepsis asociada a catéter umbilical, catéter central, catéter percutáneo, ventilación mecánica y alimentación parenteral. Expresado en $\mathrm{N}^{\mathrm{o}}$ total y tasa por mil días.

3) Análisis de Fallecidos. Recopilación en listado por columnas de los siguientes elementos de la Auditoria de Defunción actualmente en uso.

- Edad al fallecimiento.

- Peso nacimiento.

- Apgar 1 minuto.

- Apgar 5 minutos.

- Edad gestacional al nacer.

- Diagnóstico principal de patología del neonato.
- Segundo diagnóstico de importancia.

- Causa inmediata de muerte; debido a; como consecuencia de:

- Análisis causa muerte. Expresar a que grupo corresponde la causa: Prematurez no viable, anomalía congénita, problema respiratorio, infección connatal, infección nosocomial, asfixia o problema del parto, trastorno metabólico, enterocolitis necrotizante, otra causa.

- Condición o no de incompatible perse con la vida y motivo: Trisomia 13 ó 18 , decisión antenatal de no reanimar, prematurez no viable, anencefalia, hidranencefalia, Sínd. de Potter con hipoplasia pulmonar.

Se debe propender a realizar estudio anatomopatológico en todos los mortineonatos, exceptuando sólo los casos cuyo diagnóstico este absolutamente confirmado y que no sea relevante su investigación.

\section{4) Análisis de neonatos con Apgar a los 5 minutos menor o igual a 6}

Se recomienda realizar auditoria de todos los casos de neonatos con Apgar a los 5 minutos menor o igual a 6 nacidos en el servicio y determinar en el análisis la causa de éste.

Consignar en listado recopilación de los siguientes elementos de la auditoria:

- Fecha de nacimiento.

- Peso nacimiento.

- Edad gestacional al nacer.

- Apgar 1 minuto.

- Apgar 5 minutos.

- Reanimado, Si o No.

- Gases de cordón o gases < 30 minutos, consignando $\mathrm{pH}, \mathrm{pCO}_{2}$, bicarbonato y EB.

- Diagnóstico principal de patología del neonato.

- Segundo diagnóstico de importancia.

- Análisis de causa de Apgar bajo, expresado según alguna de las siguientes causas: Prematurez, hipoxia intrauterina, respiratoria, anomalía congénita, farmacológica, decisión de no reanimar, falla de reanimación, causa no precisada.

\section{5) Indicadores de Calidad en Atención Perinatal}

Además de los indicadores de IIH ya esta- 
blecidos desde nivel central y los indicadores generales de resultado expresados en el punto 1 y descrito como Informe General, debemos generar indicadores de calidad, los cuales son fundamentales en la concepción actual del quehacer médico. Se sugieren los siguientes indicadores de calidad perinatal:

- Tasa de Administración Errónea de Medicamentos (AEM): $\mathrm{N}^{\mathrm{o}}$ de eventos de AEM/ $\mathrm{N}^{\circ}$ egresos del período x 1000 .

- Tasa de Traumatismo Obstétrico Grave (TOG): Corresponde a daño por acción de fuerza sobre partes fetales que determina hospitalización y/o seguimiento al egreso de nivel superior a Atención Primaria de Salud y se expresa como: $\mathrm{N}^{0}$ casos con TOG/ $\mathrm{N}^{\mathrm{o}}$ recién nacidos del período x 1000 . Considerar aparte las fractura de clavícula.

- Tasa de Fractura de Clavícula (FC): $\mathrm{N}^{\mathrm{o}}$ casos con $\mathrm{FC} / \mathrm{N}^{\mathrm{o}}$ nacidos del período por vía vaginal x 1000

- \% Lactancia Materna Exclusiva (LME) en egresos de neonatología según grupos de peso. Expresado como $\mathrm{N}^{\mathrm{o}}$ de $\mathrm{RN}$ egresados con LME / $\mathrm{N}^{\mathrm{o}}$ egreso del período x 100. Se debe analizar por grupos de peso, $<1000 \mathrm{~g}$, 1 000-1 499 g, 1500 -1 999 g, > 1999 g.

- RN con más de 48 horas de uso de antibiótico endovenoso de segunda y tercera línea expresado en; $\mathrm{N}^{\mathrm{o}}$ total de pacientes con $\mathrm{AB}$ de $2^{\circ}$ línea/número de egresos del período $\mathrm{x} 100 \mathrm{y} \mathrm{N}^{\mathrm{o}}$ total de pacientes con $\mathrm{AB}$ de $3^{\circ}$ línea/número de egresos del período $\mathrm{x}$ 100 .

\section{Agradecimientos}

La realización de estas Recomendaciones ha pasado por diferentes etapas, siendo la inicial el desarrollo del documento al interior de la Directiva de la Rama de Neonatología de la Sociedad Chilena de Pediatría, que surge como una necesidad dada la ausencia de trabajos nacionales referidos a estos temas. Una vez terminado el primer borrador se contactó a los Jefes de Neonatología de diversos Centros Neonatales Públicos y Universitarios del país para su revisión Se obtuvo respuesta de la gran mayoría de ellos, con aportes y comentarios que han venido a enriquecer este trabajo. Agra- decemos el aporte de los profesionales que respondieron a la invitación para revisar este documento, sin cuya contribución este trabajo hubiese estado incompleto y no tendría la fuerza necesaria para ser un referente sobre Recomendaciones Neonatales.

Especial reconocimiento y agradecimiento a los profesionales del Ministerio de Salud relacionados a la Neonatología en haber revisado este documento.

La participación de estos profesionales no necesariamente representa en su totalidad la opinión personal de cada uno, sino que el Documento es un consenso del trabajo de la Directiva de la Rama de Neonatología y los aportes individuales de cada uno de los profesionales participantes.

\section{Jefes de Neonatología que participaron en la revisión:}

Arica, Dra. Leonor Schenone C. Iquique, Dr. Oscar González V. Antofagasta, Dra. Carmen Díaz Q. La Serena, Dr. Fernando Carvajal E. Valparaíso, Dra. Isabel Saldés E. Viña del Mar, Dra. Jane Standen H. San Felipe, Dr. Gastón del Solar Z. Rancagua, Dra. Carmen Gutiérrez S. Curicó, Pedro Pavez B.

Talca, Álvaro Méndez F. Concepción, Dra. Roxana Aguilar B. Chillán, Dr. Julio Salas C.

Temuco, Dra. M. Angélica Belmar S. Valdivia, Dra. Alicia Ide S.

Osorno, Dr. Carlos Brousse E. Puerto Montt, Dr. Claudio Martínez L. Coyhaique, Dra. Patricia Álvarez C. Punta Arenas, Dra. Patricia Amarales O. Santiago, SSM Oriente, Dr. Enrique Hering A. Santiago, SSM Occidente, Dra. Margarita Samamé M.

Santiago, SSM Central, Dra. Verónica Peña N. Santiago, SSM Norte, Dr. Germán Mulhausen M. Santiago, SSM Sur, Dr. Francisco Correa A. Santiago, SSM Sur, Dra. Maria Vera SM. Santiago, SSM Sur-Oriente, Dra. Patricia Mena N. Santiago, Hospital Clínico Universidad de Chile, Dr. Jaime Burgos M. Santiago, Hospital Clínico Universidad Católica de Chile, Dr. Álvaro González M. 


\section{Profesionales Colaboradores externos a Jefatura de Servicios de Neonatología:} Ministerio de Salud, Subsecretaria de Salud Pública, Dr. Carlos Becerra F.

Ministerio de Salud, Subsecretaria de Redes, Dra. Mónica Morgues N.

Ministerio de Salud, Subsecretaria de Salud

Pública, Dra. Dolores Tohá T.

Ministerio de Salud, Subsecretaria de Redes,

E.U Cecilia Reyes A.

Servicio de Salud Metropolitano Oriente,

Dr. Waldo Osorio C.

Capítulo de Enfermería Neonatal, SOCHIPE, EU Marcela Sanz R.

Capítulo de Enfermería Neonatal, SOCHIPE, Mt. María Teresa Lohse S.

\section{Referencias}

1.- Comité de Estándares y Junta Directiva de la Sociedad Española de Neonatología. Niveles asistenciales y recomendaciones de mínimos para la atención neonatal. An Pediatr (Barc) 2004; 60 (1): 54-64.

2.- García del Río M, Sánchez Luna M, Martínez D, y cols: Revisión de los estándares y recomendaciones para el diseño de una unidad de neonatología". An Pediatr (Barc) 2007; 67(6): 594-602.

3.- Comité de Estándares y Junta Directiva de la Sociedad Española de Neonatología: Niveles asistenciales y recomendaciones de mínimos para la atención neonatal". An Pediatr (Barc) 2004; 60 (1): 56-64.

4.- Guías 2004 de organización y funcionamiento de unidades de pacientes críticos. Revista Chilena de Medicina Intensiva 2004; Vol 19 (4): 209-23.

5.- White RD: Recommended standards for the newborn ICU. Journal of Perinatology (2007) 27, S4-S19.

6. Level and Volume of Neonatal Intensive Care and Mortality in Very Low Birth Weight Infants. NEJM 2007; 356: 2165-75.

7.- Are we regionalized enough? Early neonatal deaths in low risk births by the size of delivery units in Hesse, Germany 1990-1999. International Journal of Epidemiology 2002; 31: 1061-8.

8.- Hospital Volume and Neonatal Mortality among Very Low Birth Weight Infants: Pediatrics 2006; 117: 2206-14.

9.- Martin GI: Recommended Standards for Newborn ICU Design. J Perinatol 2003; 23 (Suppl 1): 1-24.

10.- Planificación de recursos y prestaciones de los servicios de Neonatología de los Hospitales de Insalud (año 2000). Dotaciones e infraestructura y aparataje de las unidades. Cartera de Servicios. Madrid: Insalud, 2002.

11.- Documento de trabajo interno no publicado de Comisión Nacional de Neonatología, Minsal, 2007 sobre Regionalización del cuidado perinatal: Recomendaciones para la acreditación de las Unidades.

12.- Guías Nacionales de Neonatología 2005. Ministerio de Salud.

13.- Guías de Atención Humanizada del proceso Reproductivo. Minsal 2007.

14.- Norma técnica básica de Autorización Sanitaria para establecimientos de Salud de Atención Cerrada. Ministerio de Salud. 2008. www.minsal.

15.- Guía de Planificación y diseño de Unidades de Cuidados Intensivos de Adultos. División de Inversiones y Desarrollo en la Red Asistencial. Ministerio de Salud 1997.

16.- División de Inversiones y Desarrollo e la Red Asistencial. Ministerio de Salud 1997.

17.- Validación de Camas Críticas en Chile. ORD $16^{\mathrm{a}}$ Nº 606711 Noviembre 2004 Subsecretaría de Salud.

18.- Guía de funcionamiento y Organización de Unidades de Cuidados Intensivos Pediátricos Chile. Subsecretaría de Redes. Comité MINSAL-Rama de Intensivo Pediátrico.

19.- Informe de Auditoria de Muerte Fetal e Infantil. MINSAL- DEIS, Departamento de Estadísticas e Información En Salud.

20.- Morgues $M$, Reyes $C$ : Informe Técnico. RN con menos de 32 semanas de edad gestacional. Sistema Nacional de Servicios de Salud de Chile. Quinquenio 20002004. MINSAL.

21.- Decreto Supremo No 57/2007, http://www.supersalud. cl/normativa/571/articles-4799_recurso_1.pdf 